\title{
THE SECOND PALOMAR SKY SURVEY
}

\author{
I.N. REID \\ Palomar Observatory \\ California Institute of Technology \\ Pasadena \\ U.S.A.
}

The 48-inch Oschin Schmidt telescope at Palomar Observatory was used to obtain the first deep, optical, wide-field survey, covering almost two-thirds of the sky between November 1949 and December 1958 (with plates for the Whiteoak extension, to $\delta_{c}=-42^{\circ}$, taken in 1964/5). The original survey was taken in two passbands - wide blue and narrow red - on 103a emulsion, and covers one hemisphere in 643 fields with a spacing of 6 degrees between centres. In 1987, plate-taking started for the second Palomar Sky Survey, POSS II, which is intended to cover the northern hemisphere in three passbands, blue (IIIa-J + GG385 filter), red (IIIa-F + RG610) and near-infrared (IVN + RG9). Given the extremely restricted overlap between fields in the old survey, we have followed the example of the UK Schmidt ESO/SERC surveys and have adopted a 5-degree spacing between fields. To date (31.7.93), we have acquired plates of acceptable quality for 597 fields (67\%) of the IIIa-J survey, 668 fields (75\%) of the IIIa-F and 253 fields (28\%) of the IVN.

Photographic copying of the POSS II plates is being undertaken by the ESO Photolabs - as is well illustrated in this volume. So far, some 510 plates have been shipped from Caltech to ESO for copying, onto glass and film. In addition, the plates are being scanned by at least two measuring machine groups - by STScI (Lasker, this volume) and by the US Naval Observatory (Monet 1993). The latter project is aimed primarily at obtaining accurate astrometry, and we have been taking short-exposure (3-minute unhypered IIIa-J) plates of each field to permit measurement of bright fundamental reference stars. The latter plates are taken within \pm 30 days of the deep IIIa-J plates to minimise uncertainties arising from proper motions. Given the current rate of progress, we expect to complete the IIIa-J and IIIa-F surveys (weather permitting) by the end of 1996, while the IVN survey is likely to extend into 1997.

In addition to support from the California Institute of Technology, POSS II has received financial support from National Geographic, the National Science Foundation, the Sloan Foundation, the Samuel Oschin Foundation and Eastman Kodak, who are also providing the photographic plate material.

\section{References}

Monet, D., 1993. ASP Conference Series, Vol. 43, ed. T. Soifer, p. 139. 